\title{
The impact of age on approach-related complications with navigated lateral lumbar interbody fusion
}

\author{
Yamaan S. Saadeh, MD, Clay M. Elswick, MD, Eleanor Smith, MD, Timothy J. Yee, MD, \\ Michael J. Strong, MD, PhD, Kevin Swong, MD, Brandon W. Smith, MD, Mark E. Oppenlander, MD, \\ Osama N. Kashlan, MD, MPH, and Paul Park, MD
}

Department of Neurosurgery, University of Michigan, Ann Arbor, Michigan

\begin{abstract}
OBJECTIVE Age is known to be a risk factor for increased complications due to surgery. However, elderly patients can gain significant quality-of-life benefits from surgery. Lateral lumbar interbody fusion (LLIF) is a minimally invasive procedure that is commonly used to treat degenerative spine disease. Recently, 3D navigation has been applied to LLIF. The purpose of this study was to determine whether there is an increased complication risk in the elderly with navigated LLIF. METHODS Patients who underwent 3D-navigated LLIF for degenerative disease from 2014 to 2019 were included in the analysis. Patients were divided into elderly and nonelderly groups, with those 65 years and older categorized as elderly. Ninety-day medical and surgical complications were recorded. Patient and surgical characteristics were compared between groups, and multivariate regression analysis was used to determine independent risk factors for complication.

RESULTS Of the 115 patients included, 56 were elderly and 59 were nonelderly. There were 15 complications (25.4\%) in the nonelderly group and $10(17.9 \%)$ in the elderly group, which was not significantly different $(p=0.44)$. On multivariable analysis, age was not a risk factor for complication $(p=0.52)$. However, multiple-level LLIF was associated with an increased risk of approach-related complication (OR 3.58, $p=0.02$ ).
\end{abstract}

CONCLUSIONS Elderly patients do not appear to experience higher rates of approach-related complications compared with nonelderly patients undergoing 3D navigated LLIF. Rather, multilevel surgery is a predictor for approach-related complication.

https://thejns.org/doi/abs/10.3171/2020.6.FOCUS20311

KEYWORDS lateral lumbar interbody fusion; three-dimensional navigation; transpsoas approach; elderly; approach complications

$\mathrm{T}$ HE lateral lumbar interbody fusion (LLIF) technique was initially described in 2006. ${ }^{1}$ Since then, its popularity and use have increased dramatically. In comparison with traditional posterior interbody fusion techniques, studies have demonstrated superiority in improved segmental lordosis and higher fusion rates. ${ }^{2} \mathrm{Al}-$ though the LLIF technique is generally considered safe, complications can occur. Well-described complications from the lateral approach include femoral nerve injuries resulting in transient or permanent weakness in hip flexion, pain or paresthesias in the thigh, neuropraxia, abdominal pseudohernias, and psoas muscle hematomas or abscesses, among others. Although uncommon, vascular and bowel injuries have also been reported..$^{3-5}$

Studies describing complications of the lateral approach are many, but few have focused on complications in the elderly (65 years or older) population. It is well established within the literature that elderly status places patients at higher risk of medical complications, including pulmonary, cardiac, and renal complications, and a higher risk of mortality. However, there is also evidence that elderly patients gain significant health-related quality of life with spine surgery, including both decompression and fusion procedures. ${ }^{6-8}$

Given the propensity of elderly patients to experience higher operative morbidity and mortality, the unique risk profile of lateral approaches to lumbar interbody fusion, and the relative scarcity of literature on this topic in relation to age, we conducted a retrospective review of patients undergoing navigated LLIF at our institution to assess for differences in complication occurrence in elderly compared with nonelderly patients. 


\section{Methods}

\section{Surgical Technique}

The technique utilized has been previously described..$^{9,10}$ To briefly summarize, after induction of general anesthesia, the patient was placed in the lateral decubitus position (most frequently left side up). An axillary roll was placed under the patient, and then a large gel roll was placed just cephalad to the iliac crest to facilitate opening the costopelvic interval. The patient was then securely taped to the operating table. Attention was taken to ensure that an ample portion of the anterior and posterior superior iliac spines were exposed for fixation of the navigation frame.

The patient was then prepped and draped in the usual fashion. An iliac pin was fixed into the iliac crest posterior to the anterior superior iliac spine for placement of the navigation reference frame. An intraoperative CT scanner was then brought into the field for thin-cut CT imaging capture of the target levels.

Next, spinal navigation was used to plan an entry point on the flank, marking the anterior and posterior portions of the disc. An incision was made, and cautery was used to expose to the external abdominal muscle fascia. The abdominal musculature was then opened using blunt dissection, avoiding the use of cautery, to decrease the risk of abdominal pseudohernia due to a nerve injury. Using a long hemostat, the fascia over the external oblique muscle was then opened, and dissection was continued through the internal oblique muscle, transversalis fascia, and transverse abdominal fascia to access the retroperitoneal space. After visualizing retroperitoneal fat, finger dissection is performed to palpate the quadratus lumborum posteriorly, the psoas muscle, and the lumbar transverse processes on the lateral aspect of the spine. The kidney is often palpable anteriorly at the upper lumbar levels.

At this point, a navigated dilator connected to a stimulator lead was used to traverse the anterior aspect of the psoas muscle to land on the midpoint of the target disc space and puncture the annulus. If no significant electromyographic activity was present on monitoring, the dilator tubes were sequentially placed, and a self-retaining retractor was fixed into position. An annulotomy was created, discectomy was performed, and the endplate was prepared with the assistance of navigated instruments. An appropriately sized cage was inserted with navigation guidance. After LLIF, patients typically underwent repositioning to the prone position for posterior surgery, which included pedicle screw fixation.

\section{Data Collection}

With institutional review board approval, the medical records of all patients who underwent LLIF between June 2014 and January 2019 were retrospectively reviewed. Inclusion criteria included use of intraoperative CT image acquisition and 3D computer-assisted spinal navigation for LLIF. Patients who had undergone a prior anterior or lateral approach to the lumbar spine were excluded. Complications were defined as any medical or surgical adverse events that occurred within 90 days postoperatively. Complication data collected included sensory and/or motor deficits, ileus, infection, CSF leak, and cardiac and pul-
TABLE 1. Patient characteristics

\begin{tabular}{lccc}
\hline & Elderly $(n=56)$ & Nonelderly $(n=59)$ & $p$ Value \\
\hline Mean age, yrs & $71.9 \pm 4.9$ & $57.4 \pm 6.2$ & $<0.0001$ \\
\hline Female sex & $33(58.9)$ & $37(62.7)$ & 0.68 \\
\hline Mean BMI & $29.2 \pm 5.5$ & $29.9 \pm 6.2$ & 0.50 \\
\hline Current smoker & $3(5.3)$ & $11(18.6)$ & 0.054 \\
\hline Multiple levels treated & $24(42.9)$ & $30(50.9)$ & 0.39 \\
\hline Treatment at L4-5 & $31(55.4)$ & $41(69.5)$ & 0.12 \\
\hline
\end{tabular}

Values represent the number of patients (\%) unless stated otherwise. Mean values are presented as the mean \pm SD.

monary complications. All complications regardless of approach were collected. Patients were divided based on age into elderly patients (age $\geq 65$ years) and nonelderly patients (age $<65$ years).

\section{Statistical Analysis}

A chi-square analysis for categorical variables and an independent t-test for continuous variables were performed to analyze for differences between groups in terms of demographics (age, sex, BMI, and smoking status), number of levels treated, and treatment at L4-5. A multivariate logistic regression model was then used to control for potential confounding variables, including sex, BMI, smoking status, single level versus multiple levels treated, and whether treatment included the L4-5 level. Statistical analysis was performed using SAS software (SAS Institute Inc.).

\section{Results}

One hundred fifteen consecutive patients were included in the analysis. There were 56 elderly patients and 59 nonelderly patients (Table 1). The average age of the elderly cohort was 71.9 years. The average age of the nonelderly group was 57.4 years. The average length of follow-up was 13.3 months. BMI was similar for the two groups: $29.2 \mathrm{~kg} / \mathrm{m}^{2}$ in the elderly group and $29.9 \mathrm{~kg} / \mathrm{m}^{2}$ in the nonelderly group. The nonelderly group had a higher percentage of patients who were smokers than the elderly group, although this was not a statistically significant difference.

In the elderly group, 7 patients (12.5\%) experienced transient sensory symptoms, and 10 patients (17.9\%) experienced transient mild motor deficits (Table 2). In the nonelderly group, 12 patients (20.3\%) experienced transient sensory symptoms, and 7 patients $(11.9 \%)$ experienced transient mild motor deficits. These symptoms resolved completely by 3 months postoperatively.

At the 3-month follow-up, the elderly group had 4 patients $(7.1 \%)$ who experienced persistent sensory symptoms and 3 patients (5.4\%) who experienced persistent mild motor deficits. In the nonelderly group, 3 patients (5.1\%) experienced persistent sensory symptoms, and 1 patient $(1.8 \%)$ experienced persistent mild motor deficits. Of the elderly patients with persistent motor weakness, 2 of them were graded as having $4+/ 5$ hip flexor strength at the 3-month 
TABLE 2. Complication frequency

\begin{tabular}{|c|c|c|c|}
\hline & \multicolumn{2}{|c|}{ No. of Patients (\%) } & \multirow[b]{2}{*}{ p Value } \\
\hline & Elderly & Nonelderly & \\
\hline Transient sensory symptoms & $7(12.5)$ & $12(20.3)$ & 0.55 \\
\hline Transient motor deficit & $10(17.9)$ & $7(11.9)$ & 0.18 \\
\hline $\begin{array}{l}\text { Persistent sensory symptoms at } \\
3 \text { mos }\end{array}$ & $4(7.1)$ & $3(5.1)$ & 0.66 \\
\hline Persistent motor deficit at 3 mos & $3(5.4)$ & $1(1.7)$ & 0.28 \\
\hline lleus & $1(1.8)$ & $5(8.5)$ & 0.11 \\
\hline Infection & $0(0)$ & $2(3.4)$ & 0.57 \\
\hline Superficial dehiscence & $0(0)$ & $1(1.7)$ & 0.59 \\
\hline CSF leak & $0(0)$ & $1(1.7)$ & 0.59 \\
\hline Clostridium difficile colitis & $0(0)$ & $1(1.7)$ & 0.59 \\
\hline Pulmonary complication & $1(1.8)$ & $1(1.7)$ & 0.97 \\
\hline Cardiac complication & $1(1.8)$ & $0(0)$ & 0.59 \\
\hline $\begin{array}{l}\text { Total no. of complications (ex- } \\
\text { cluding transient symptoms) }\end{array}$ & $10(17.9)$ & $15(25.4)$ & 0.44 \\
\hline
\end{tabular}

follow-up, and the third was graded as having 4/5 hip flexor strength. The nonelderly patient with persistent motor deficit was graded as having 4+/5 hip flexor strength at the 3 -month follow-up. At the 1-year follow-up, 2 of these patients had full resolution of their mild weakness.

Other complications in the elderly group included $1 \mathrm{pa}-$ tient with ileus $(1.8 \%), 1$ patient with a cardiac complication $(1.8 \%)$, and 1 patient with a pulmonary complication $(1.8 \%)$. Other complications in the nonelderly group included 5 patients with ileus (8.5\%), 2 patients with infection (3.4\%), 1 patient with superficial wound dehiscence (1.7\%), 1 patient with a CSF leak (1.7\%), and 1 patient with a pulmonary complication (1.7\%). The CSF leak and wound dehiscence occurred with the posterior surgical approach.

In total, within the elderly group 10 patients $(17.9 \%)$ experienced complications in the perioperative period. In the nonelderly group, 15 patients $(25.4 \%)$ experienced a perioperative complication. Notably, transient sensory and transient motor symptoms were not included as complications.

Multivariate logistic regression analysis also did not demonstrate a statistically significant difference in complications in elderly patients compared with nonelderly patients after controlling for BMI, sex, smoking status, treatment at L4-5, and treatment of single versus multiple levels (Table 3).

On further analysis, our multivariate logistic regression model did show that treatment of multiple levels compared with treatment at a single level was associated with a statistically significant increased risk of approach-related complication (OR 3.58, $\mathrm{p}=0.02)$. Multivariate analysis did not show any change in complications related to sex, BMI, smoking status, or treatment at L4-5.

\section{Discussion}

To our knowledge, this is the first study of its size on
TABLE 3. Multivariate analysis of complications

\begin{tabular}{lccc}
\hline & OR & $95 \% \mathrm{Cl}$ & $\mathrm{p}$ Value \\
\hline Age $\geq 65$ yrs & 0.72 & $0.26-1.97$ & 0.52 \\
\hline BMI & 0.96 & $0.86-1.05$ & 0.33 \\
\hline Male vs female & 1.09 & $0.41-2.92$ & 0.87 \\
\hline Current smoker vs nonsmoker & 1.51 & $0.32-7.04$ & 0.91 \\
\hline Former smoker vs nonsmoker & 2.67 & $0.90-7.88$ & 0.14 \\
\hline Multiple levels treated vs single & 3.58 & $1.24-10.29$ & 0.02 \\
level treated & & & \\
\hline Treatment at L4-5 & 0.52 & $0.19-1.42$ & 0.20 \\
\hline
\end{tabular}

age-related complications of navigated LLIF. The results of our study suggest that patients aged 65 years and older who undergo lateral interbody fusion are not at higher risk than patients younger than 65 years for approach-related complications. Rather than age, multilevel interbody fusion was a significant risk factor for increased complication occurrence.

With regard to our analysis, we elected not to include transient sensory and motor symptoms in the total complications results. The lateral retroperitoneal technique by definition results in traversing the psoas muscle, resulting in an expectation of postoperative proximal lower-extremity pain that can result in mild pain-limited weakness. Additionally, some degree of transient sensory symptoms can be expected, given the potential retraction of the sensory branches of the lumbar plexus during the approach. Of the persistent motor symptoms, the severity was mild, and 2 of the 4 patients fully recovered at 1 year.

Although no complication was found to be significantly higher between groups on univariate analysis, ileus in particular approached significance $(p=0.11)$. Notably, $8.5 \%$ of nonelderly patients experienced ileus postoperatively compared with $1.8 \%$ of elderly patients. It is possible a larger study would show a meaningful difference.

Multivariate analysis found that the treatment of multiple levels compared with a single level was statistically significant for a higher rate of complication, with an OR greater than 3 . This finding is potentially related to the increased operative time, including time of psoas retraction, and the number of times the psoas is traversed when more than 1 level is treated. It is also likely that the underlying condition was more complex, necessitating a multilevel operation.

There have been a number of studies on approach-related complications from LLIF. Knight et al. ${ }^{11}$ reported on 58 patients undergoing traditional, nonnavigated LLIF. In that series, 5 patients had transient sensory symptoms, 1 patient had persistent sensory symptoms, 5 patients had transient motor deficit, 2 patients had persistent motor deficit, and 3 patients experienced medical complications. Moller et al. ${ }^{12}$ reported on 53 patients undergoing traditional LLIF. Of these patients, 21 had transient sensory symptoms, 9 had persistent sensory symptoms, 16 had transient motor deficits, and 3 had persistent motor deficits. Januszewski et al. ${ }^{13}$ reported on 61 patients undergoing traditional LLIF; 9 patients had transient sensory symptoms, 1 patient had 
persistent sensory symptoms, 0 patients had transient motor deficits, and 2 patients had persistent motor deficits. Le et al ${ }^{14}$ reported on 71 patients undergoing traditional LLIF; 11 patients had transient sensory symptoms, 3 patients had persistent sensory symptoms, 39 patients had transient motor deficits, and 0 patients had persistent motor deficits. Cummock et al. ${ }^{15}$ reported on 59 patients undergoing traditional LLIF; 30 patients had transient sensory symptoms, 7 patients had persistent sensory symptoms, 14 patients had transient motor deficits, 0 patients had persistent motor deficits, 3 patients required wound revisions, and 1 patient had a medical complication. The overall complication profile in this study is consistent with the literature.

The study has several limitations. This is a single-institution study, limiting generalizability given the differences among institutions and techniques. Data collection was also retrospective, so there exists the potential for underreporting of complications.

\section{Conclusions}

In this large, retrospective review of elderly and nonelderly patients undergoing LLIF, older age was not an independent risk factor for development of an approachrelated complication. Rather, treatment of more than 1 level was predictive of an approach-related complication.

\section{References}

1. Ozgur BM, Aryan HE, Pimenta L, Taylor WR. Extreme Lateral Interbody Fusion (XLIF): a novel surgical technique for anterior lumbar interbody fusion. Spine J. 2006;6(4): 435-443.

2. Saadeh YS, Joseph JR, Smith BW, et al. Comparison of segmental lordosis and global spinopelvic alignment after single-level lateral lumbar interbody fusion or transforaminal lumbar interbody fusion. World Neurosurg. 2019;126:e1374e1378.

3. Uribe JS, Deukmedjian AR. Visceral, vascular, and wound complications following over 13,000 lateral interbody fusions: a survey study and literature review. Eur Spine J. 2015; 24(suppl 3):386-396.

4. Kueper J, Fantini GA, Walker BR, et al. Incidence of vascular complications during lateral lumbar interbody fusion: an examination of the mini-open access technique. Eur Spine J. 2015;24(4):800-809.

5. Balsano M, Carlucci S, Ose M, Boriani L. A case report of a rare complication of bowel perforation in extreme lateral interbody fusion. Eur Spine J. 2015;24(suppl 3):405-408.

6. Cloyd JM, Acosta FL Jr, Ames CP. Complications and outcomes of lumbar spine surgery in elderly people: a review of the literature. J Am Geriatr Soc. 2008;56(7):1318-1327.

7. Jakola AS, Sørlie A, Gulati S, et al. Clinical outcomes and safety assessment in elderly patients undergoing decompressive laminectomy for lumbar spinal stenosis: a prospective study. BMC Surg. 2010;10:34.
8. Drazin D, Shirzadi A, Rosner J, et al. Complications and outcomes after spinal deformity surgery in the elderly: review of the existing literature and future directions. Neurosurg Focus. 2011;31(4):E3.

9. Joseph JR, Smith BW, Patel RD, Park P. Use of 3D CT-based navigation in minimally invasive lateral lumbar interbody fusion. J Neurosurg Spine. 2016;25(3):339-344.

10. Park P. Three-dimensional computed tomography-based spinal navigation in minimally invasive lateral lumbar interbody fusion: feasibility, technique, and initial results. Neurosurgery. 2015;11(suppl 2):259-267.

11. Knight RQ, Schwaegler P, Hanscom D, Roh J. Direct lateral lumbar interbody fusion for degenerative conditions: early complication profile. J Spinal Disord Tech. 2009;22(1): 34-37.

12. Moller DJ, Slimack NP, Acosta FL Jr, et al. Minimally invasive lateral lumbar interbody fusion and transpsoas approach-related morbidity. Neurosurg Focus. 2011;31(4):E4.

13. Januszewski J, Vivas AC, Bach K, et al. Minimally invasive lateral transpsoas interbody fusion at the $\mathrm{L} 4 / 5$ level: a review of 61 consecutive cases. Oper Neurosurg (Hagerstown). 2018; 15(4):447-453.

14. Le TV, Burkett CJ, Deukmedjian AR, Uribe JS. Postoperative lumbar plexus injury after lumbar retroperitoneal transpsoas minimally invasive lateral interbody fusion. Spine (Phila Pa 1976). 2013;38(1):E13-E20.

15. Cummock MD, Vanni S, Levi AD, et al. An analysis of postoperative thigh symptoms after minimally invasive transpsoas lumbar interbody fusion. J Neurosurg Spine. 2011; 15(1):11-18.

\section{Disclosures}

Dr. Oppenlander: consultant for Globus, DePuy Spine, and LifeNet Health. Dr. Park: consultant for Globus and NuVasive; royalties from Globus; and support of non-study-related clinical or research effort from ISSG and DePuy.

\section{Author Contributions}

Conception and design: Park, Saadeh. Acquisition of data: Saadeh, Elswick, E Smith. Analysis and interpretation of data: Park, Saadeh, Elswick. Drafting the article: Saadeh, Elswick. Critically revising the article: Park, Saadeh, Yee, Strong, BW Smith. Reviewed submitted version of manuscript: all authors. Approved the final version of the manuscript on behalf of all authors: Park. Statistical analysis: Kashlan. Administrative/ technical/material support: Park, Saadeh. Study supervision: Park, Kashlan.

\section{Correspondence}

Paul Park: University of Michigan, Ann Arbor, MI. ppark@med. umich.edu. 